\title{
Local Fractional Discrete Wavelet Transform for Solving Signals on Cantor Sets
}

\author{
Yang Zhao, ${ }^{1,2}$ Dumitru Baleanu, ${ }^{3,4,5}$ Carlo Cattani, ${ }^{6}$ De-Fu Cheng, ${ }^{1}$ and Xiao-Jun Yang ${ }^{7}$ \\ ${ }^{1}$ College of Instrumentation \& Electrical Engineering, Jilin University, Changchun 130061, China \\ ${ }^{2}$ Electronic and Information Technology Department, Jiangmen Polytechnic, Jiangmen 529090, China \\ ${ }^{3}$ Department of Chemical and Materials Engineering, Faculty of Engineering, King Abdulaziz University, P.O. Box 80204, \\ Jeddah 21589, Saudi Arabia \\ ${ }^{4}$ Department of Mathematics and Computer Sciences, Faculty of Arts and Sciences, Cankaya University, 06530 Ankara, Turkey \\ ${ }^{5}$ Institute of Space Sciences, Magurele, 077125 Bucharest, Romania \\ ${ }^{6}$ Department of Mathematics, University of Salerno, Via Ponte don Melillo, Fisciano, 84084 Salerno, Italy \\ ${ }^{7}$ Department of Mathematics and Mechanics, China University of Mining and Technology, Xuzhou Campus, Xuzhou, \\ Jiangsu 221008, China
}

Correspondence should be addressed to De-Fu Cheng; chengdefu@jlu.edu.cn

Received 15 September 2013; Accepted 22 October 2013

Academic Editor: J. A. Tenreiro Machado

Copyright (C) 2013 Yang Zhao et al. This is an open access article distributed under the Creative Commons Attribution License, which permits unrestricted use, distribution, and reproduction in any medium, provided the original work is properly cited.

The discrete wavelet transform via local fractional operators is structured and applied to process the signals on Cantor sets. An illustrative example of the local fractional discrete wavelet transform is given.

\section{Introduction}

In recent years, the classical wavelet theory [1-7] has played an important role in many scientific fields such as signal processing [8], electrical systems [9], image processing [10], and differential equations [11]. The continuous wavelet transform is applied to handle the analyzing nonstationary signals, which have some characteristics of instantaneous peaks or discontinuities, where the mother wavelet met scaling and translation operations [3]. Two major categories of wavelet transforms are continuous and discrete [5]. When the mother wavelet functions are orthonormal, the discrete wavelet transform [12] gives multiresolution algorithm decomposing signals into scales with different time and frequency resolution, which leads to finite number of wavelet comparisons of signals, and improves the computational speeds because of the functions that are stretched or compressed and placed at many positions along the signals [13].

Based on the fractional Fourier transform [14-17], the fractional wavelet transform, which was a good tool for processing transient signals and compressing images, was structured in $[18,19]$. The fractional wavelet transform has some applications in various branches of science and engineering [20-23]. For example, the simultaneous spectral analysis of a binary mixture system was presented in [20] by using the fractional wavelet transform. Application of the fractional wavelet transform to the simultaneous determination of ampicillin sodium and sulbactam sodium in a binary mixture was considered in [21]. The fractional wavelet transform for the quantitative spectral resolution of the composite signals of the active compounds in a twocomponent mixture was suggested in [22]. The optical image encryption based on fractional wavelet transform was given in [23]. By discretizing continuous fractional wavelet transform, the discrete fractional wavelet transform was reported and its application to multiple encryptions was considered in [24].

The wavelet method and its fractional counterpart have many applications in various branches of science and engineering. However, they are invalid for solving the signals 
defined on Cantor sets. The local fractional calculus theory [25-34] was applied to handle the functions defined on Cantor sets, which are local fractional continuous. A natural question is to generalize signals concepts on the Cantor set, which are the nondifferentiable functions defined on Cantor sets $[24,26]$ and the Cantor function [35]. The mathematical theory of the local fractional wavelet transform of the local fractional continuous signal was structured in $[25,36]$ based on the basic idea.

One of the open problems in this area is how to improve the computational speeds of the local fractional wavelet theory as in the classical one. The aim of this paper is to structure the discrete version of the local fractional wavelet transform based on the generalized inner production space. The paper has been organized as follows. In Section 2, we introduce some basic notations and theorems of the generalized inner product space. In Section 3, we propose the local fractional discrete wavelet transform. In Section 4, one example is presented. Finally, Section 5 is conclusions.

\section{Preliminaries}

In this section, we give some basic notations and theorems of the generalized inner product space.

Let [25]

$$
\begin{gathered}
L_{2, \alpha}[R]=\left\{f(x) \in C_{\alpha}[R]:\left(\frac{1}{\Gamma(1+\alpha)} \int_{-\infty}^{\infty}|f(x)|^{p}(d x)^{\alpha}\right)^{1 / p}\right. \\
<\infty, 1 \leq p<\infty\} .
\end{gathered}
$$

Here, the local fractional integral operator $f(x)$ in the interval $[a, b]$ was defined in $[25-30]$ as

$$
\begin{aligned}
a_{b}^{(\alpha)} f(x) & =\frac{1}{\Gamma(1+\alpha)} \int_{a}^{b} f(t)(d t)^{\alpha} \\
& =\frac{1}{\Gamma(1+\alpha)} \lim _{\Delta t \rightarrow 0} \sum_{j=0}^{j=N-1} f\left(t_{j}\right)\left(\Delta t_{j}\right)^{\alpha},
\end{aligned}
$$

where a partition of the interval $[a, b]$ is denoted as $\Delta t_{j}=$ $t_{j+1}-t_{j}, \Delta t=\max \left\{\Delta t_{0}, \Delta t_{1}, \Delta t_{j}, \ldots\right\}$ and $j=0, \ldots, N-1$, $t_{0}=a, t_{N}=b$. Local fractional operators were applied to model some nondifferentiable problems [25-32].

From (1) the generalized inner product space of $L_{2, \alpha}[R]$ is defined as follows [25]:

$$
\langle f, g\rangle_{\alpha}=\frac{1}{\Gamma(1+\alpha)} \int_{-\infty}^{\infty} f(x) \overline{g(x)}(d x)^{\alpha}
$$

The two useful theorems are presented as follows.
Theorem 1 (see [25]). Let $X$ be an inner product space. If $\left\{e_{n}^{\alpha}\right\}$ is an orthonormal system in $X$, then one has that

$$
\begin{gathered}
\|f\|_{\alpha}^{2}=\sum_{i=1}^{\infty}\left|\left\langle f, e_{i}^{\alpha}\right\rangle_{\alpha}\right|^{2}, \\
f=\sum_{i=1}^{\infty}\left\langle f, e_{i}^{\alpha}\right\rangle_{\alpha} e_{i}^{\alpha}
\end{gathered}
$$

are equivalent, where $\|f\|_{\alpha}^{2}$ is a norm of the function $f$ and $\left\{e_{n}^{\alpha}\right\}$ has the following properties:

$$
\begin{gathered}
\left\|e_{n}^{\alpha}\right\|_{\alpha}=1, \\
\left\langle e_{i}^{\alpha}, e_{j}^{\alpha}\right\rangle= \begin{cases}0, & i \neq j, \\
1, & i=j .\end{cases}
\end{gathered}
$$

Proof. See [25].

Theorem 2 (see [25]). Let $X$ be an inner product space and $\left\{e_{n}^{\alpha}\right\}$ be an orthonormal system in $X$. If $x^{\alpha} \in \operatorname{span}\left\{e_{1}^{\alpha}, \ldots, e_{n}^{\alpha}\right\}$, then for all $x^{\alpha} \in X$ one has

$$
x^{\alpha}=\sum_{i=1}^{n}\left\langle x^{\alpha}, e_{i}^{\alpha}\right\rangle_{\alpha} e_{i}^{\alpha},
$$

where $\operatorname{span}\left\{x_{1}^{\alpha}, \ldots, x_{n}^{\alpha}\right\}$ is the linear subspace of $X$ of the linear span of the local fractional vectors [25], namely,

$$
\operatorname{span}\left\{x_{1}^{\alpha}, \ldots, x_{n}^{\alpha}\right\}=\left\{x^{\alpha}=\sum_{i=1}^{n} a_{i} x_{i}^{\alpha}: a_{i} \in E\right\}
$$

Proof. See [25].

\section{Local Fractional Discrete Wavelet Transform for Signals on Cantor Sets}

3.1. Local Fractional Continuous Wavelet Transformation for Signals on Cantor Sets. The local fractional continuous wavelet transform of the local fractional continuous signal $f(t)$ was presented in $[25,26,36]$ as

$$
\begin{array}{r}
W_{\varphi, \alpha} f(a, b)=\frac{a^{-(\alpha / 2)}}{\Gamma(1+\alpha)} \int_{-\infty}^{\infty} f(t) \overline{\varphi_{a, b, \alpha}(t)}(d t)^{\alpha}, \\
0<\alpha \leq 1,
\end{array}
$$

where the local fractional daughter's wavelets were suggested in $[25,26,36]$ by

$$
\varphi_{a, b, \alpha}(t)=\frac{1}{a^{\alpha / 2}} \varphi\left(\frac{t-b}{a}\right),
$$

where $a$ is the dyadic dilation, $b$ is the dyadic position, and $a^{-(\alpha / 2)}$ is the normalization Cantor factor. The inverse 


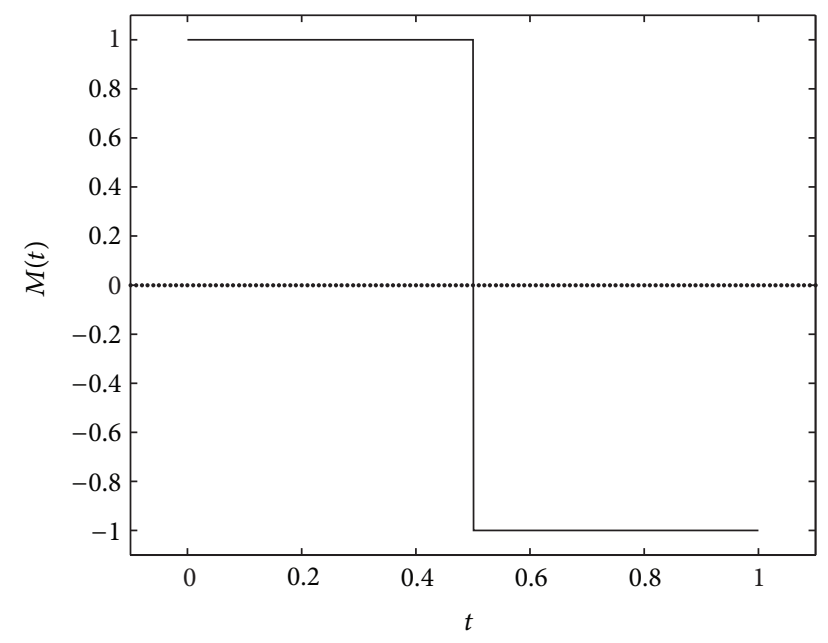

FIgURE 1: The graph of the local fractional mother wavelet.

formula of local fractional wavelet transform was given in $[25,36]$ by

$$
\begin{aligned}
f(x)= & \frac{C_{\varphi, \alpha}}{\Gamma^{2}(1+\alpha)} \\
& \times \int_{-\infty}^{\infty} \int_{-\infty}^{\infty} a^{-2 \alpha} W_{\varphi, \alpha} f(a, b) \varphi_{a, b, \alpha}(t)(d a)^{\alpha}(d b)^{\alpha}, \\
& 0<\alpha \leq 1,
\end{aligned}
$$

where the parameter is $[25,36]$

$$
C_{\varphi, \alpha}=\frac{1}{\Gamma(1+\alpha)} \int_{-\infty}^{\infty} \frac{|f(x)|^{2}}{|x|^{\alpha}}(d x)^{\alpha}, \quad 0<\alpha \leq 1 .
$$

We notice that the classical continuous wavelet transform is the local fractional one in case of fractal dimension $\alpha=1$.

3.2. Local Fractional Discrete Wavelet Transform for Signals on Cantor Sets. Let us structure the local fractional daughter wavelet in the form

$$
\varphi_{a, b, \alpha}(t)=\frac{1}{a^{\alpha / 2}} \varphi\left(\frac{t-b}{a}\right),
$$

where $\varphi \in L_{2, \alpha}[R]$.

$$
\begin{aligned}
& \text { When } a=2^{-j} \text { and } b=k 2^{-j} \text {, we get } \\
& \varphi_{a, b, \alpha}(t)=\varphi_{j, k, \alpha}(t)=\varphi_{2^{-j}, k 2^{-j}, \alpha}(t)=2^{j \alpha / 2} \varphi\left(2^{j} t-k\right)
\end{aligned}
$$

for integers $j, k \in \mathrm{Z}$.

Let $\varphi_{j, k, \alpha}(t)=2^{j \alpha / 2} \varphi\left(2^{j} t-k\right)$ be orthogonal set of local fractional wavelets. Then we can obtain

$$
\left\langle\varphi_{j, k, \alpha}, \varphi_{m, n, \alpha}\right\rangle_{\alpha}=\delta_{j, m}^{\alpha} \delta_{k, n}^{\alpha}, \quad j, k, m, n \in \mathrm{Z},
$$

where $\delta_{j, m}^{\alpha}$ and $\delta_{k, n}^{\alpha}$ are local fractional Kronecker delta [27].

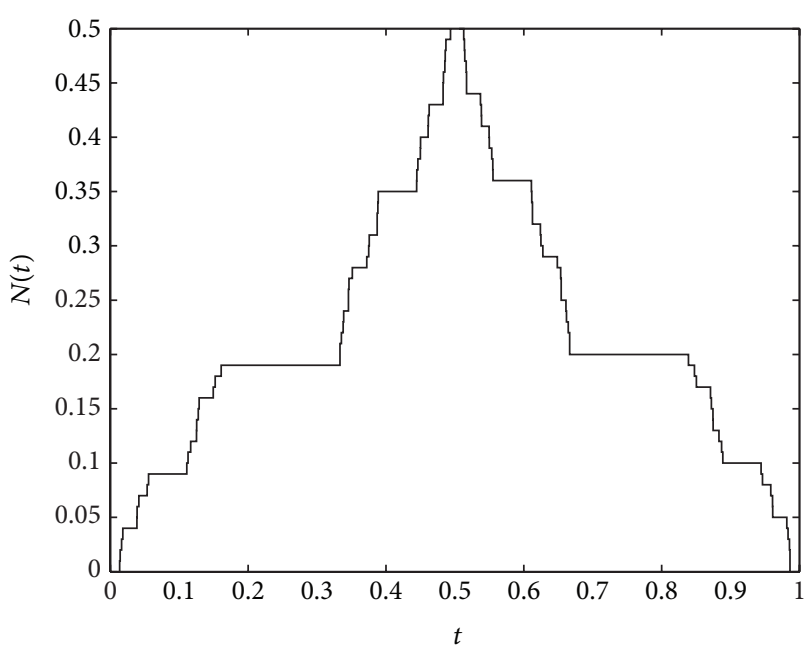

FIGURE 2: The graph of the local fractional integral of local fractional mother wavelet.

Making use of (7), for $j, k, m \in \mathrm{Z}$ we have

$$
f(x)=\sum_{j=-\infty}^{\infty} \sum_{m=-\infty}^{\infty} a_{j, k, \alpha} e_{j, k}^{\alpha}
$$

where its coefficients are

$$
a_{j, k}=\left\langle f(x), e_{j, k}^{\alpha}\right\rangle_{\alpha}=W_{\varphi, \alpha} f\left(2^{-j}, k 2^{-j}\right) .
$$

Here, $a_{j, k}$ is called as the local fractional discrete wavelet transform of the signal $f(x)$.

\section{An Illustrative Example}

Local fractional mother wavelet is defined in [26] as

$$
\varphi_{H(\alpha)}(t)=M(t)= \begin{cases}1, & 0 \leq t<\frac{1}{2} \\ -1, & \frac{1}{2} \leq t<1 \\ 0, & \text { else }\end{cases}
$$

and local fractional integral of local fractional mother wavelet reads as

$$
\phi_{H(\alpha)}(t)=N(t)= \begin{cases}\frac{t^{\alpha}}{\Gamma(1+\alpha)}, & 0 \leq t<\frac{1}{2} \\ \frac{(1-t)^{\alpha}}{\Gamma(1+\alpha)}, & \frac{1}{2} \leq t<1 \\ 0, & \text { else. }\end{cases}
$$

Figure 1 shows the graph of the local fractional mother wavelet and Figure 2 shows the graph of the local fractional integral of local fractional mother wavelet.

When fractal dimension $\alpha=1$, we have

$$
\varphi_{H(1)}(t)=M(t)
$$




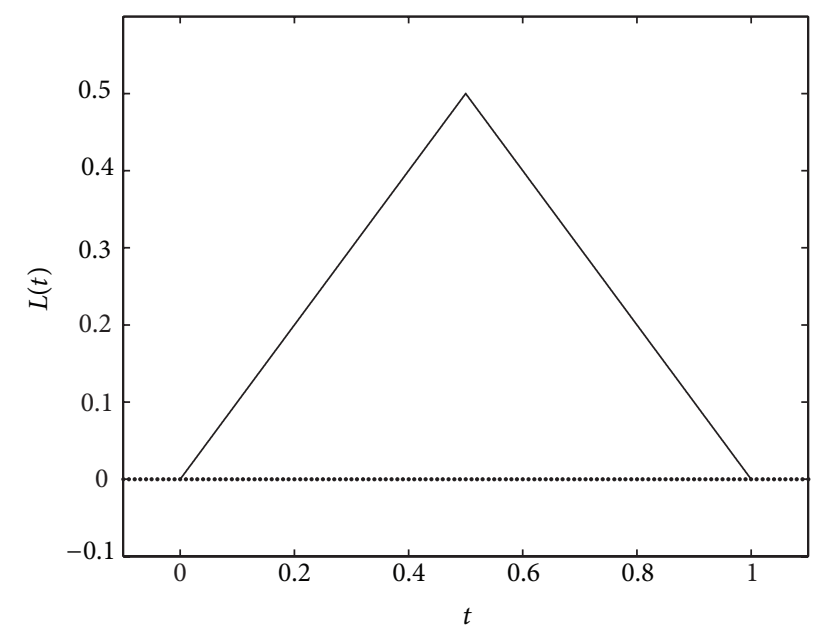

Figure 3: The graph of the integral of the mother wavelet.

so that

$$
\phi_{H(1)}(t)=L(t)= \begin{cases}t, & 0 \leq t<\frac{1}{2} \\ 1-t, & \frac{1}{2} \leq t<1 \\ 0, & \text { else. }\end{cases}
$$

Figure 3 shows the graph of the integral of mother wavelet $\varphi_{H(1)}(t)$.

For integers $j, k \in \mathrm{Z}$, we have [26]

$$
\varphi_{H(\alpha)}^{j, k}(t)=2^{j \alpha / 2} \varphi_{H(\alpha)}\left(2^{j} t-k\right),
$$

where

$$
\varphi_{H(\alpha)}(t)= \begin{cases}1, & 0 \leq t<\frac{1}{2} \\ -1, & \frac{1}{2} \leq t<1 \\ 0, & \text { else. }\end{cases}
$$

Hence, we have

$$
\begin{aligned}
\left\langle\varphi_{H(\alpha)}^{j, k}, \varphi_{H(\alpha)}^{m, n}\right\rangle_{\alpha} & \frac{1}{\Gamma(1+\alpha)} \int_{-\infty}^{\infty} \varphi_{H(\alpha)}^{j, k}(t) \varphi_{H(\alpha)}^{m, n}(t)(d t)^{\alpha} \\
= & \frac{1}{\Gamma(1+\alpha)} \\
& \times \int_{-\infty}^{\infty} 2^{j \alpha / 2} \varphi_{H(\alpha)}\left(2^{j} t-k\right) 2^{m \alpha / 2} \varphi_{H(\alpha)} \\
= & 2^{(j+m) \alpha / 2} \frac{1}{\Gamma(1+\alpha)} \\
& \times \int_{-\infty}^{\infty} \varphi_{H(\alpha)}\left(2^{j} t-k\right)(d t)^{\alpha} \\
= & 2^{(m-j) \alpha / 2} \frac{1}{\Gamma(1+\alpha)} \varphi_{H(\alpha)}\left(2^{m} t-n\right)(d t)^{\alpha} \\
& \times \int_{-\infty}^{\infty} \varphi_{H(\alpha)}(s) \varphi_{H(\alpha)}\left(2^{m-j}(s+k)-n\right)(d s)^{\alpha},
\end{aligned}
$$

where $s=2^{j} t-k$.
In view of (24), we obtain [15]

$$
\begin{gathered}
\frac{1}{\Gamma(1+\alpha)} \int_{-\infty}^{\infty}\left[\varphi_{H(\alpha)}^{j, k}(t)\right]^{2}(d t)^{\alpha}=1, \\
\frac{1}{\Gamma(1+\alpha)} \int_{-\infty}^{\infty} \varphi_{H(\alpha)}^{j, k}(t)(d t)^{\alpha}=0,
\end{gathered}
$$

where $j=m$ and $k=n, j, k \in \mathrm{Z}$.

When $j=m, j, k, m \in Z$, from (24) we obtain

$$
\left\langle\varphi_{H(\alpha)}^{j, k}, \varphi_{H(\alpha)}^{j, n}\right\rangle_{\alpha}
$$$$
=\frac{1}{\Gamma(1+\alpha)} \int_{-\infty}^{\infty} \varphi_{H(\alpha)}^{j, k}(t) \varphi_{H(\alpha)}^{m, n}(t)(d t)^{\alpha}
$$$$
=2^{(j+m) \alpha / 2} \frac{1}{\Gamma(1+\alpha)}
$$$$
\times \int_{-\infty}^{\infty} \varphi_{H(\alpha)}\left(2^{j} t-k\right) \varphi_{H(\alpha)}\left(2^{m} t-n\right)(d t)^{\alpha}
$$$$
=2^{(m-j) \alpha / 2} \frac{1}{\Gamma(1+\alpha)}
$$

$$
\begin{aligned}
& \times \int_{-\infty}^{\infty} \varphi_{H(\alpha)}(s) \varphi_{H(\alpha)}\left(2^{m-j}(s+k)-n\right)(d s)^{\alpha} \\
= & \frac{1}{\Gamma(1+\alpha)} \\
& \times \int_{-\infty}^{\infty} \varphi_{H(\alpha)}(s) \varphi_{H(\alpha)}(s+k-n)(d s)^{\alpha} \\
= & \delta_{0, k-n}^{\alpha} \\
= & \delta_{k, n}^{\alpha}
\end{aligned}
$$

where $s=2^{j} t-k$.

When $g=m-j>0, j, k, m, n \in \mathrm{Z}$, from (24) we have

$$
\begin{aligned}
\left\langle\varphi_{H(\alpha)}^{j, k}, \varphi_{H(\alpha)}^{m, n}\right\rangle_{\alpha} & \frac{1}{\Gamma(1+\alpha)} \int_{-\infty}^{\infty} \varphi_{H(\alpha)}^{j, k}(t) \varphi_{H(\alpha)}^{m, n}(t)(d t)^{\alpha} \\
= & 2^{g \alpha / 2} \frac{1}{\Gamma(1+\alpha)} \\
& \times \int_{-\infty}^{\infty} \varphi_{H(\alpha)}(s) \varphi_{H(\alpha)}\left(2^{g}(s+k)-n\right)(d s)^{\alpha} \\
= & 2^{g \alpha / 2} \frac{1}{\Gamma(1+\alpha)} \\
& \times \int_{-\infty}^{\infty} \varphi_{H(\alpha)}(s) \varphi_{H(\alpha)}\left(2^{g} s+\eta\right)(d s)^{\alpha},
\end{aligned}
$$


where $s=2^{j} t-k$ and $\eta=2^{g} k-n$. Consider

$$
\begin{aligned}
& \left\langle\varphi_{H(\alpha)}^{j, k}, \varphi_{H(\alpha)}^{m, n}\right\rangle_{\alpha} \\
& =2^{g \alpha / 2} \frac{1}{\Gamma(1+\alpha)} \\
& \quad \times \int_{-\infty}^{\infty} \varphi_{H(\alpha)}(s) \varphi_{H(\alpha)}\left(2^{g} s+\eta\right)(d s)^{\alpha} \\
& =2^{g \alpha / 2} \frac{1}{\Gamma(1+\alpha)} \\
& \quad \times \int_{-\infty}^{\infty} \varphi_{H(\alpha)}(s) \varphi_{H(\alpha)}\left(2^{g} s+\eta\right)(d s)^{\alpha} \\
& =2^{g \alpha / 2}\left[\frac{1}{\Gamma(1+\alpha)} \int_{0}^{1 / 2} \varphi_{H(\alpha)}\left(2^{g} s+\eta\right)(d s)^{\alpha}\right. \\
& \left.\quad-\frac{1}{\Gamma(1+\alpha)} \int_{1 / 2}^{1} \varphi_{H(\alpha)}\left(2^{g} s+\eta\right)(d s)^{\alpha}\right] \\
& =2^{-g \alpha / 2}\left[\frac{1}{\Gamma(1+\alpha)} \int_{\eta}^{2^{g-1}+\eta} \varphi_{H(\alpha)}(q)(d q)^{\alpha}\right. \\
& \left.\quad 2_{2^{g-1}+\eta}^{2^{g}+\eta} \varphi_{H(\alpha)}(q)(d q)^{\alpha}\right],
\end{aligned}
$$

where

$$
\begin{gathered}
q=2^{g} s+\eta, \\
\frac{1}{\Gamma(1+\alpha)} \int_{\eta}^{2^{g-1}+\eta} \varphi_{H(\alpha)}(q)(d q)^{\alpha}=0, \\
\frac{1}{\Gamma(1+\alpha)} \int_{2^{g-1}+\eta}^{2^{g}+\eta} \varphi_{H(\alpha)}(q)(d q)^{\alpha}=0,
\end{gathered}
$$

with $\eta>1,2^{g-1}+\eta>1$, and $2^{g}+\eta>1$.

Hence, taking $e_{j, k}^{\alpha}=\varphi_{H(\alpha)}^{j, k}$ gives

$$
f(x)=\sum_{j=-\infty}^{\infty} \sum_{m=-\infty}^{\infty} a_{j, k, \alpha} \varphi_{H(\alpha)}^{j, k}(x),
$$

where

$$
\begin{aligned}
a_{j, k} & =\left\langle f(x), \varphi_{H(\alpha)}^{j, k}(x)\right\rangle_{\alpha} \\
& =W_{\varphi, \alpha} f\left(2^{-j}, k 2^{-j}\right) \\
& =2^{j \alpha / 2} \frac{1}{\Gamma(1+\alpha)} \int_{-\infty}^{\infty} f(x) \bar{\varphi}_{H(\alpha)}^{j, k}(x)(d x)^{\alpha} .
\end{aligned}
$$

Appling (4), we have

$$
f^{2}(x)=\sum_{i=1}^{\infty}\left|a_{j, k}\right|^{2}
$$

with

$$
a_{j, k}=2^{j \alpha / 2} \frac{1}{\Gamma(1+\alpha)} \int_{-\infty}^{\infty} f(x) \bar{\varphi}_{H(\alpha)}^{j, k}(x)(d x)^{\alpha} .
$$

Hence, from (32) we find that the energy is conserved.

\section{Conclusions}

In this work the local fractional discrete wavelet transform based on the local fractional calculus theory was proposed. By using the basic theorems of generalized inner product space, the local fractional discrete wavelet transform and its reconstruction formula were discussed. We find that the energy of the signal on Cantor sets is conserved. An illustrative example for the local fractional wavelet transform of the signal on Cantor sets was given. It is shown that the classical discrete wavelet transform is the local fractional one in case of fractal dimension $\alpha=1$.

\section{Conflict of Interests}

The authors declare that they have no conflict of interests regarding this paper.

\section{Acknowledgment}

The authors would like to thank the referees for their useful comments and remarks. $s$

\section{References}

[1] I. Daubechies, Ten Lectures on Wavelets, vol. 61 of CBMS-NSF Regional Conference Series in Applied Mathematics, Society for Industrial and Applied Mathematics (SIAM), Philadelphia, Pa, USA, 1992.

[2] D. Baleanu, Advances in Wavelet Theory and Their Applications in Engineering, Physics and Technology, InTech, Rijeka, Croatia, 2012.

[3] I. Daubechies, "The wavelet transform, time-frequency localization and signal analysis," IEEE Transactions on Information Theory, vol. 36, no. 5, pp. 961-1005, 1990.

[4] A. Grossmann and J. Morlet, "Decomposition of Hardy functions into square integrable wavelets of constant shape," SIAM Journal on Mathematical Analysis, vol. 15, no. 4, pp. 723-736, 1984.

[5] C. E. Heil and D. F. Walnut, "Continuous and discrete wavelet transforms," SIAM Review, vol. 31, no. 4, pp. 628-666, 1989.

[6] C. Cattani, "Shannon wavelets theory," Mathematical Problems in Engineering, vol. 2008, Article ID 164808, 24 pages, 2008.

[7] X.-G. Xia and Z. Zhang, "On sampling theorem, wavelets, and wavelet transforms," IEEE Transactions on Signal Processing, vol. 41, no. 12, pp. 3524-3535, 1993.

[8] O. Rioul and M. Vetterli, "Wavelets and signal processing," IEEE Signal Processing Magazine, vol. 8, no. 4, pp. 14-38, 1991.

[9] S.-J. Huang, T.-M. Yang, and J.-T. Huang, "FPGA realization of wavelet transform for detection of electric power system disturbances," IEEE Transactions on Power Delivery, vol. 17, no. 2, pp. 388-394, 2002.

[10] A. M. Wink and J. B. T. M. Roerdink, "Denoising functional MR images: a comparison of wavelet denoising and Gaussian smoothing," IEEE Transactions on Medical Imaging, vol. 23, no. 3, pp. 374-387, 2004.

[11] C. Cattani, "Harmonic wavelets towards the solution of nonlinear PDE," Computers \& Mathematics with Applications, vol. 50, no. 8-9, pp. 1191-1210, 2005. 
[12] M. J. Shensa, "The discrete wavelet transform: wedding the atrous and Mallat algorithms," IEEE Transactions on Signal Processing, vol. 40, no. 10, pp. 2464-2482, 1992.

[13] M. Bahoura and H. Ezzaidi, "FPGA-implementation of discrete wavelet transform with application to signal denoising," Circuits, Systems, and Signal Processing, vol. 31, no. 3, pp. 987-1015, 2012.

[14] D. Mendlovic and H. M. Ozaktas, "Fractional Fourier transforms and their optical implementation. I," Journal of the Optical Society of America A, vol. 10, no. 9, pp. 1875-1881, 1993.

[15] L. B. Almeida, "Fractional fourier transform and timefrequency representations," IEEE Transactions on Signal Processing, vol. 42, no. 11, pp. 3084-3091, 1994.

[16] J. Tenreiro MacHado, F. B. Duarte, and G. Monteiro Duarte, "Analysis of financial data series using fractional Fourier transform and multidimensional scaling," Nonlinear Dynamics, vol. 65, no. 3, pp. 235-245, 2011.

[17] D. Baleanu, K. Diethelm, E. Scalas, and J. J. Trujillo, Fractional Calculus Models and Numerical Methods, vol. 3 of Series on Complexity, Nonlinearity and Chaos, World Scientific, Hackensack, NJ, USA, 2012.

[18] D. Mendlovic, Z. Zalevsky, D. Mas, J. García, and C. Ferreira, "Fractional wavelet transform," Applied Optics, vol. 36, no. 20, pp. 4801-4806, 1997.

[19] M. Unser and T. Blu, "Fractional splines and wavelets," SIAM Review, vol. 42, no. 1, pp. 43-67, 2000.

[20] E. Dinç, E. Büker, and D. Baleanu, "Fractional and continuous wavelet transforms for the simultaneous spectral analysis of a binary mixture system," Communications in Nonlinear Science and Numerical Simulation, vol. 16, no. 12, pp. 4602-4609, 2011.

[21] E. Dinç and D. Baleanu, "A new fractional wavelet approach for the simultaneous determination of ampicillin sodium and sulbactam sodium in a binary mixture," Spectrochimica Acta A, vol. 63, no. 3, pp. 631-638, 2006.

[22] E. Dinç and D. Baleanu, "Fractional wavelet transform for the quantitative spectral resolution of the composite signals of the active compounds in a two-component mixture," Computers \& Mathematics with Applications, vol. 59, no. 5, pp. 1701-1708, 2010.

[23] L. Chen and D. Zhao, "Optical image encryption based on fractional wavelet transform," Optics Communications, vol. 254, no. 4-6, pp. 361-367, 2005.

[24] G. Bhatnagar, Q. M. J. Wu, and B. Raman, "Discrete fractional wavelet transform and its application to multiple encryption," Information Sciences, vol. 223, pp. 297-316, 2013.

[25] X. J. Yang, Local Fractional Functional Analysis and Its Applications, Asian Academic Publisher, Hong Kong, 2011.

[26] X. J. Yang, D. Baleanu, H. M. Srivastava, and J. A. Tenreiro Machado, "On local fractional continuous wavelet transform," Abstract and Applied Analysis, vol. 2013, Article ID 725416, 5 pages, 2013.

[27] X. J. Yang, Advanced Local Fractional Calculus and Its Applications, World Science, New York, NY, USA, 2012.

[28] C. F. Liu, S. S. Kong, and S. J. Yuan, "Reconstructive schemes for variational iteration method within Yang-Laplace transform with application to fractal heat conduction problem," Thermal Science, vol. 17, no. 3, pp. 715-721, 2013.

[29] A. M. Yang, Y. Z. Zhang, and Y. Long, "The Yang-Fourier transforms to heat-conduction in a semi-infinite fractal bar," Thermal Science, vol. 17, no. 3, pp. 707-713, 2013.
[30] X. J. Yang, D. Baleanu, and J. A. T. Machado, "Mathematical aspects of Heisenberg uncertainty principle within local fractional Fourier analysis," Boundary Value Problems, no. 1, pp. 131146, 2013

[31] X.-J. Yang, H. M. Srivastava, J.-H. He, and D. Baleanu, "Cantortype cylindrical-coordinate method for differential equations with local fractional derivatives," Physics Letters A, vol. 377, no. 28-30, pp. 1696-1700, 2013.

[32] X.-J. Yang, D. Baleanu, and J. A. Tenreiro Machado, "Systems of Navier-Stokes equations on Cantor sets," Mathematical Problems in Engineering, vol. 2013, Article ID 769724, 8 pages, 2013.

[33] A. Carpinteri, B. Chiaia, and P. Cornetti, "The elastic problem for fractal media: basic theory and finite element formulation," Computers and Structures, vol. 82, no. 6, pp. 499-508, 2004.

[34] A. K. Golmankhaneh, V. Fazlollahi, and D. Baleanu, "Newtonian mechanics on fractals subset of real-line," Romania Reports in Physics, vol. 65, pp. 84-93, 2013.

[35] O. Dovgoshey, O. Martio, V. Ryazanov, and M. Vuorinen, "The Cantor function," Expositiones Mathematicae, vol. 24, no. 1, pp. $1-37,2006$.

[36] X. J. Yang, "Local fractional calculus and its applications," in Proceedings of the 5th IFAC Workshop Fractional Differentiation and Its Applications (FDA '12), pp. 1-8, Nanjing, China, 2012. 


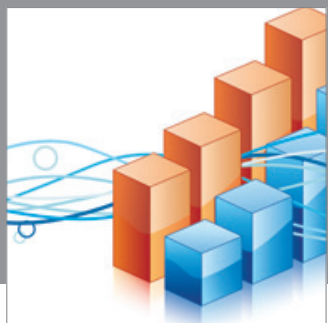

Advances in

Operations Research

mansans

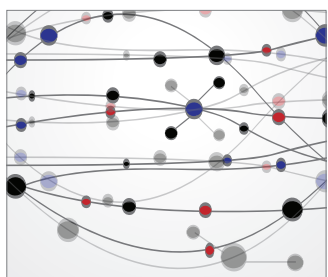

The Scientific World Journal
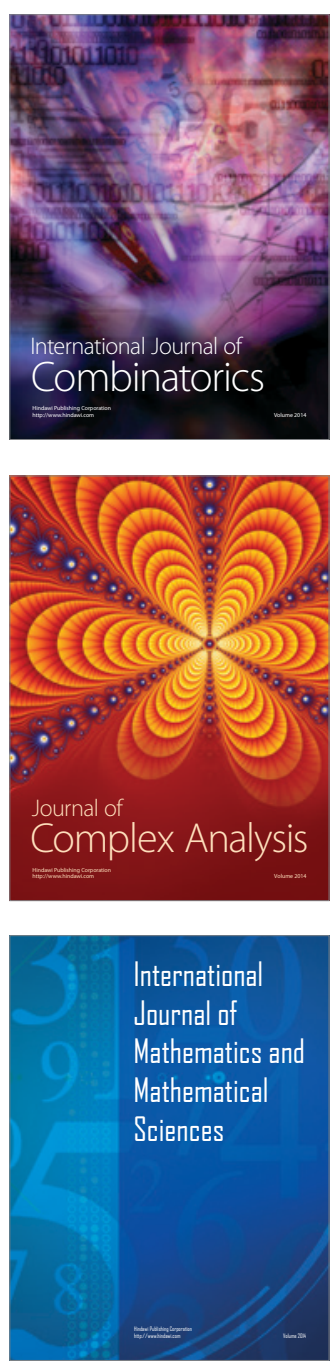
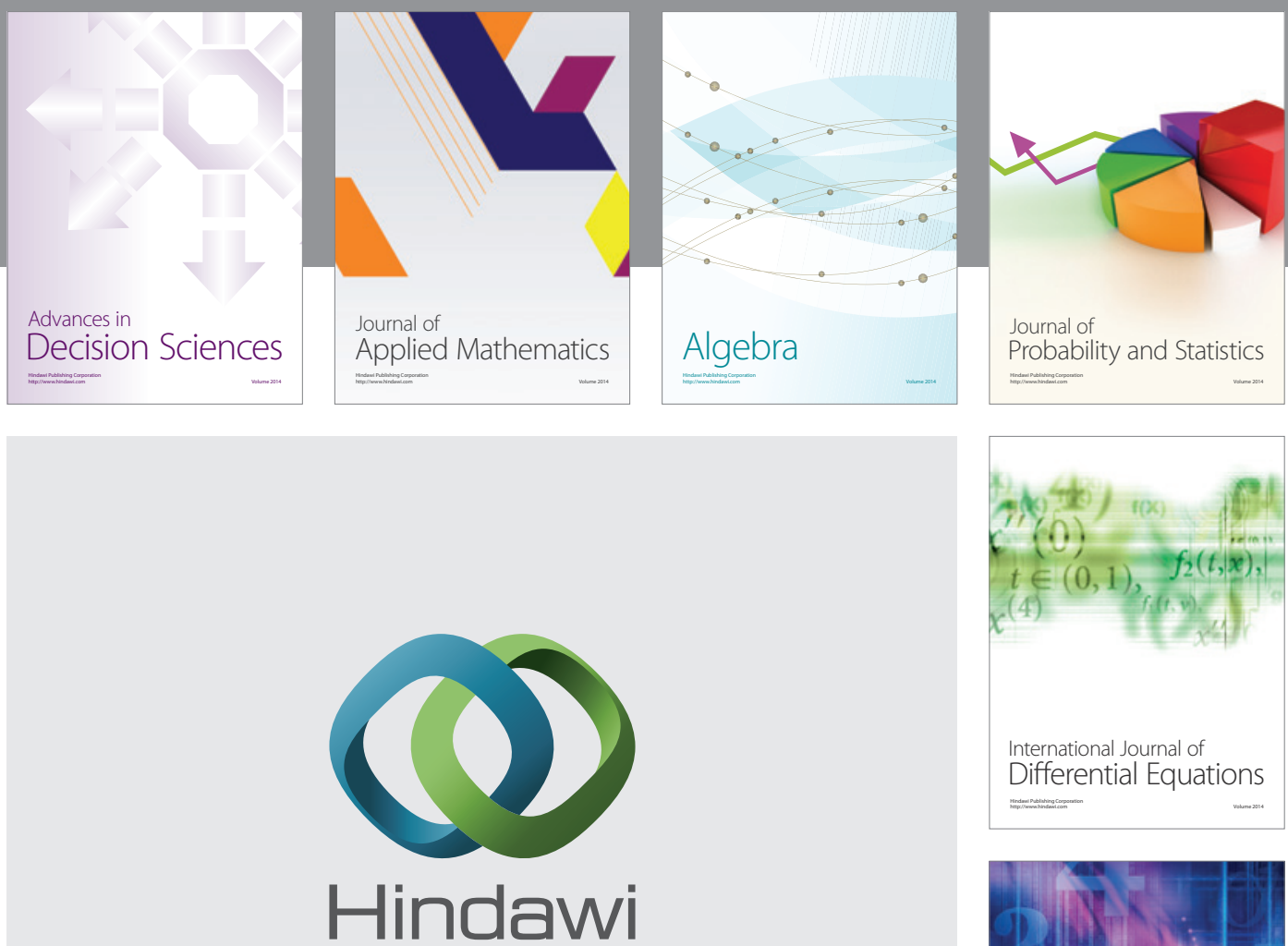

Submit your manuscripts at http://www.hindawi.com
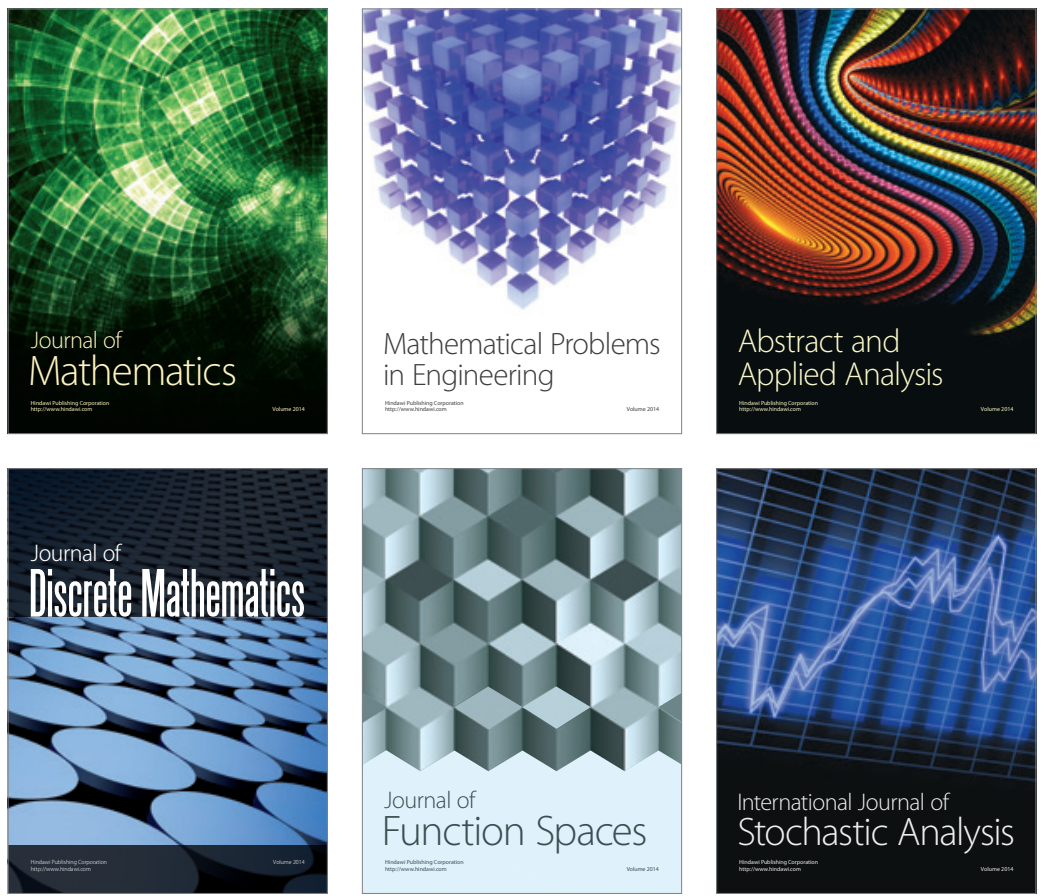

Journal of

Function Spaces

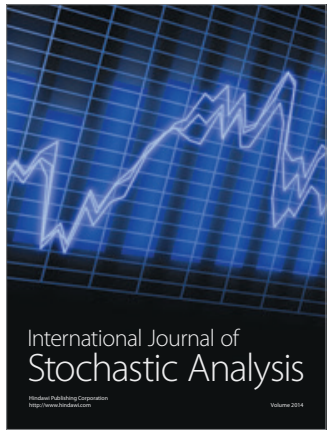

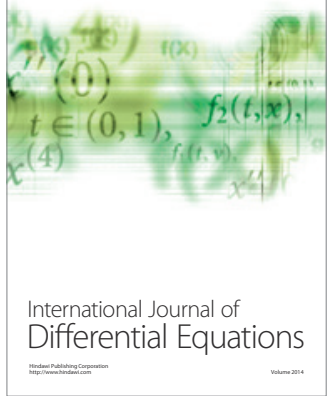
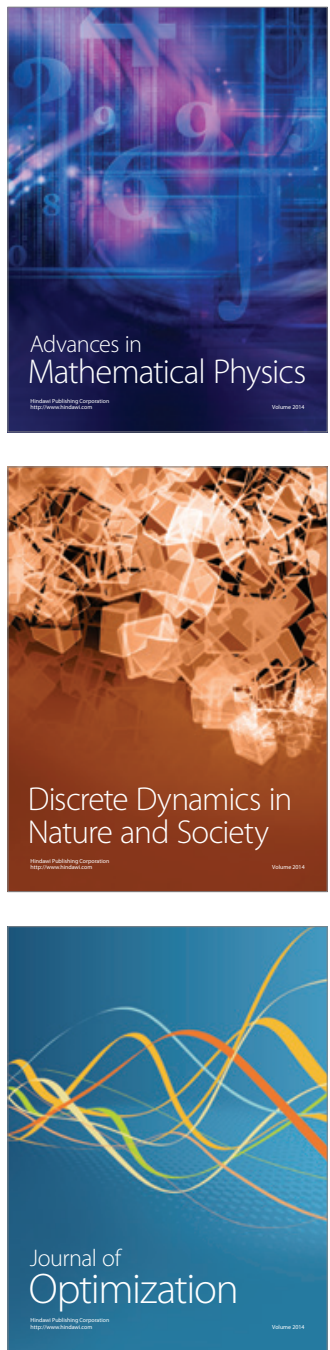